\title{
Thymic tumors and immune checkpoint inhibitors
}

\author{
Shintaro Yokoyama ${ }^{1}$, Hiroaki Miyoshi ${ }^{2}$ \\ ${ }^{1}$ Department of Surgery, ${ }^{2}$ Department of Pathology, Kurume University School of Medicine, Kurume, Japan \\ Contributions: (I) Conception and design: All authors; (II) Administrative support: None; (III) Provision of study materials or patients: All authors; (IV) \\ Collection and assembly of data: S Yokoyama; (V) Data analysis and interpretation: S Yokoyama; (VI) Manuscript writing: All authors; (VII) Final \\ approval of manuscript: All authors. \\ Correspondence to: Hiroaki Miyoshi, MD, PhD. Department of Pathology, Kurume University School of Medicine, 67 Asahi-machi, Kurume 8300011, \\ Japan. Email: miyoshi_hiroaki@med.kurume-u.ac.jp.
}

\begin{abstract}
Thymoma and thymic carcinoma, known as the most common features of thymic epithelial tumors (TETs), are thoracic malignancies displaying varied clinical features and prognosis. These neoplasms being frequently ineligible for surgical complete resection as a curative treatment because of extensive tumor spread, effectual nonsurgical treatments are needed; however, an optimal chemotherapeutic regimen has not been identified, although some regimens have been shown to be active. Immunotherapy is effective for other malignancies and may be promising as a therapeutic alternative for refractory TETs. Thus far, several studies have determined the expression of programmed death ligand 1 (PD-L1) and programmed death 1 (PD-1) in TETs, including its clinicopathological and prognostic significance. The results have been conflicting due to the different immunohistochemical antibodies employed and distinct cutoff values. However, many authors identified abundant PD-L1 expression in TETs, which is considered as an important predictive factor for therapeutic effect of PD-1 inhibitors in other malignant tumors. In some clinical trials, an acceptable clinical efficacy of PD-1 inhibitor for TETs has been reported as expected; however, concerns regarding immunological adverse events have been raised. To optimize these therapeutic agents for refractory TETs, additional studies which evaluate clinical availabilities of immunotherapeutic drugs and characterize their basic mechanisms of action against immunotherapeutic targets are both urgently required.
\end{abstract}

Keywords: Thymic tumor; thymic epithelial tumor (TET); tumor immunotherapy; programmed death ligand 1 (PD-L1); programmed death 1 (PD-1)

Submitted Oct 19, 2017. Accepted for publication Oct 25, 2017.

doi: $10.21037 /$ jtd.2017.10.157

View this article at: http://dx.doi.org/10.21037/jtd.2017.10.157

\section{Introduction}

Thymic tumors are rare neoplasms originating from the thymus and distinctly exhibit generically indolent behaviors. Thymic epithelial tumors (TETs) represented by thymoma and thymic carcinoma are the most common features in this category (1). Complete surgical resection is the standard treatment for TETs (2-6); however, treatment is often challenging for clinicians because these tumors commonly invade adjacent structures or metastasize to distant organs. In these unresectable cases, multidisciplinary systemic chemotherapy or chemoradiotherapy is administered. The initial regimens include cisplatin/doxorubicin/ cyclophosphamide with or without prednisolone, cisplatin/ doxorubicin/vincristine/cyclophosphamide (ADOC), or cisplatin/etoposide for thymoma and carboplatin/paclitaxel or ADOC in thymic carcinoma $(7,8)$. Nevertheless, the clinical efficacy of these agents is confirmed only by the results of some retrospective studies or phase II trials, and evidence from randomized controlled trial is lacking probably due to the rarity of the disease. Indeed, unsatisfied chemotherapeutic responses are often observed in clinical settings, particularly in the treatment of thymic carcinoma. Therefore, further improved treatment strategies based on solid oncogenic mechanisms are currently investigated.

Recent accumulating evidences on immune checkpoint 
Table 1 Profiles of PD-L1 expression in thymic epithelial tumors

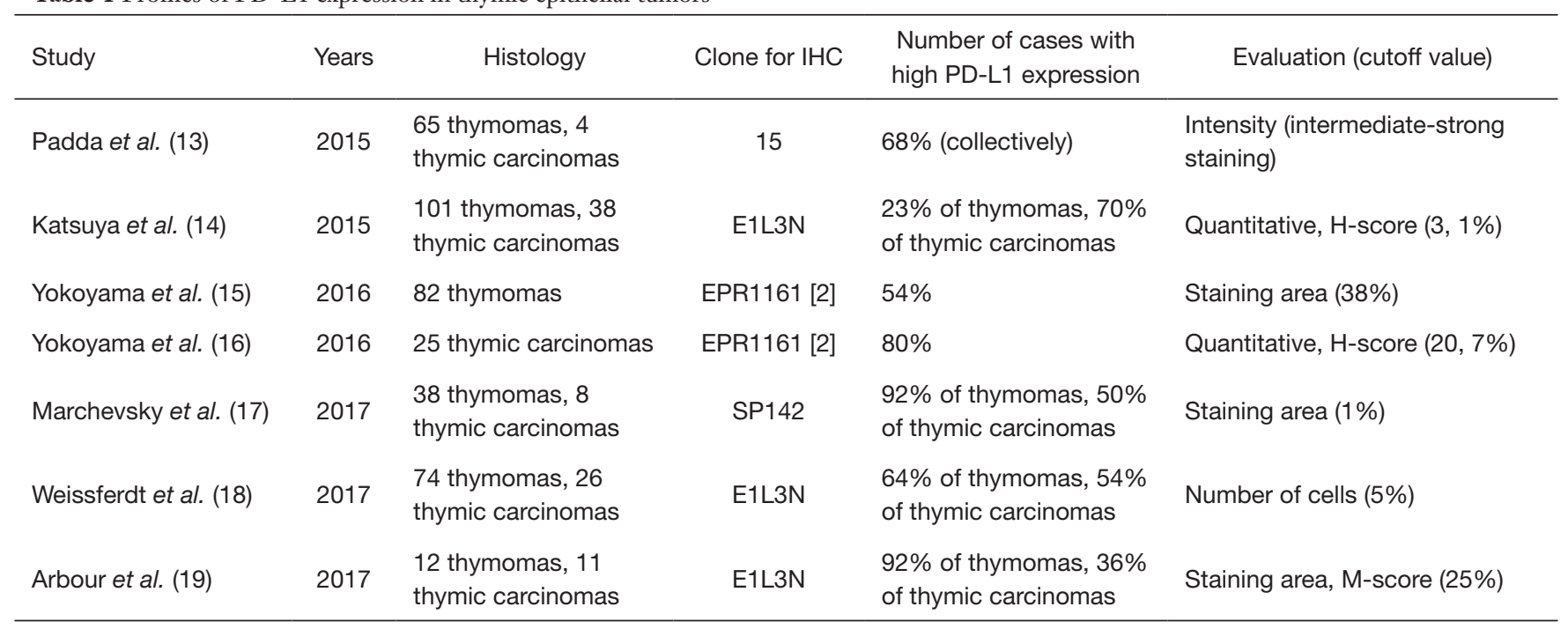

PD-L1, programmed death ligand 1; IHC, immunohistochemistry.

pathways enhanced the understanding of underlying mechanisms in tumor immunity. Programmed death ligand 1 (PD-L1) is a transmembrane glycoprotein expressed by antigen-presenting cells and modulates immune responses by binding to programmed death 1 (PD-1) receptor, a member of the $\mathrm{B} 7$ receptor family expressing on the surface of $\mathrm{T}$ cells $(9,10)$. This sequence contributes inhibitory signals that mediate physiologic immune tolerance (11). Moreover, recent studies emphasized the role of PD-1/ PD-L1 pathway in facilitating tumor progression by inhibiting anti-tumor $T$ cell responses to evade host immune surveillance mechanisms (12). Therefore, immune checkpoint inhibitors, which are novel therapeutic options for patients with malignant tumors, are effective against malignancies and have been widely used since their introduction. Several clinical trials showed the efficacy of anti-PD-1, anti-PD-L1, and anti-cytotoxic T-lymphocyte antigen-4 (CTLA-4) antibodies, leading to their approval for clinical application in various malignant tumors by the U.S. Food and Drug Administration (FDA).

In TETs, some research identified the expression of immunotherapeutic targets in the tumor microenvironment, and other case reports also indicated novel antitumor responses from the agents targeting PD-1/PD-L1 pathway. Some phase I trials assessing the effectiveness of immune checkpoint inhibitors in solid malignant tumors included a small number of TETs and confirmed their effectiveness in each case. In addition, other phase II clinical trials on PD-1/PD-L1 pathway blockers for TETs are being currently performed.
In this review article, we aimed to provide an overview on the current knowledge of immunotherapeutic targets focused on PD-L1 and PD-1 and discuss the potential for immune checkpoint blockade therapy in TETs.

\section{PD-L1 expression of neoplastic cells in TETs}

Analyses of PD-L1 expression in TETs started in 2015 following the development of PD-L1 evaluation in various malignant tumors. However, only seven studies by far have examined PD-L1 expression in TETs, 4 of which are from the United States, while the other 3 are from Japan (Table 1). However, these studies included only a limited number of cases maybe since TETs are relatively rare malignant tumors and data are difficult to collect even in cancer centers.

Padda et al. first announced PD-L1 expression in 69 TETs evaluated via immunohistochemistry (IHC) using a clone of 15 (Sino Biological, Beijing, China) (13). They identified diffuse IHC staining in neoplastic cells in almost all PD-L1-positive cases with both cell membrane and cytoplasm. PD-L1 expression status was defined according to IHC staining intensity and the proportion was not considered. They concluded that $68 \%$ of the 69 TET cases showed high PD-L1 expression. Katsuya et al. reported that $23 \%$ of thymomas and $70 \%$ of thymic carcinomas have high PD-L1 expression by evaluating using $\mathrm{H}$-score method with a cutoff value of $1 \%$, which consider both staining proportion and intensity. They employed a clone of E1L3N (Cell Signaling Technology, Danvers, MA, USA), 

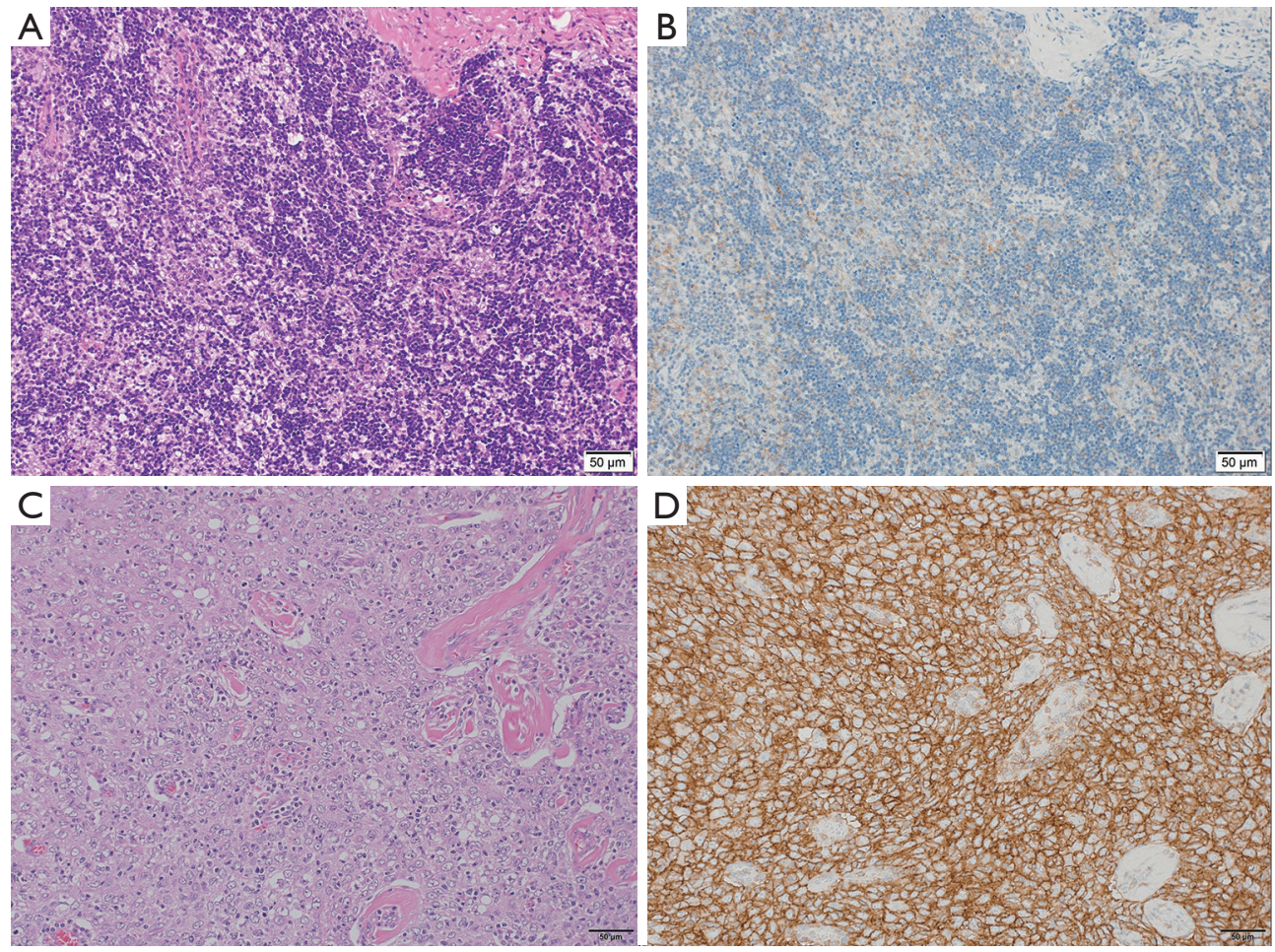

Figure 1 Representative microphotographs of immunohistochemical detection of PD-L1 in type B1 thymoma (A,B) and thymic carcinoma (C,D). Hematoxylin and eosin staining (left column, original magnification, 200x) and PD-L1 immunostaining (right column, original magnification, 200x). Diffuse staining with weak/moderate and high intensity of PD-L1 is identified in type B1 thymoma (B) and thymic carcinoma (D), respectively.

which is a high reliable antibody (14). The authors reported that $54 \%$ of thymomas and $80 \%$ of thymic carcinomas overexpressed PD-L1 $(15,16)$. PD-L1 was immunostained by using a clone of EPR1161 [2] (Abcam, Cambridge, UK), and evaluated considering staining area and $\mathrm{H}$-score method, respectively. The optimal cutoff value was validated by using receiver operating characteristic curve and Youden's index. Meanwhile, Marchevsky et al. used a SP142 clone to investigate 38 thymomas and eight thymic carcinomas and found that $92 \%$ of thymomas and $50 \%$ of thymic carcinomas exhibit high-PD-L1 expression (17). They defined the cutoff value as $1 \%$ in each area. Weissferdt et al. surveyed 100 cases of TET (74 thymomas and 26 thymic carcinomas). PD-L1 was evaluated according to the number of tumor cells showing positive staining for E1L3N (18). The cutoff value was defined as the proportion of positive cells with $5 \%$ staining. They found that $64 \%$ of thymomas and $54 \%$ of thymic carcinomas have high PD-L1 expression. Arbour et al. recently studied 12 thymomas and 11 thymic carcinomas by using E1L3N clone and evaluated
PD-L1 expression according to staining area (M-score) (19). They considered the cutoff value as $25 \%$ in $\mathrm{M}$-score and concluded that $92 \%$ of thymomas and $36 \%$ of thymic carcinomas revealed PD-L1 overexpression.

Although these studies evaluated the degree of PDL1 expression of tumor cells via IHC using varied antibodies and cutoff values, a similar result has been obtained, indicating that neoplastic epithelial cells in TETs demonstrated relatively abundant PD-L1 expression (Figure 1). In other malignancies, PD-1- and PD-L1-based antibodies showed enhanced activity, particularly in PDL1-positive tumors (20,21). Therefore, these results support the potential clinical efficacy of PD-1/PD-L1 blockade in TETs, although the practical application of such treatment modality is still under investigation in clinical trials.

\section{Clinicopathological and prognostic relevance of PD-L1 expression in TETs}

Previous studies also showed some clinicopathological 
Table 2 Clinicopathological features and prognostic significance associated with PD-L1 expression in thymic epithelial tumors

\begin{tabular}{lll}
\hline Studies & Factors associated with high PD-L1 expression & Prognostic impact of high PD-L1 expression \\
\hline Padda et al. (13) & $\begin{array}{l}\text { Younger age, high stage, incomplete resection, aggressive } \\
\text { histology }\end{array}$ & Worse OS (age- and sex-adjusted analysis) \\
Katsuya et al. (14) & Thymic carcinoma (rather than thymoma) & None \\
Yokoyama et al. (15) & Masaoka stage III/IV, WHO type B2/B3 & Worse DFS \\
Yokoyama et al. (16) & None & Better OS \\
Marchevsky et al. (17) & Aggressive histology & - \\
Weissferdt et al. (18) & Neoadjuvant therapy in thymoma & None \\
Arbour et al. (19) & Thymoma (rather than thymic carcinoma) & Better OS
\end{tabular}

PD-L1, programmed death ligand 1; WHO, World Health Organization; OS, overall survival; DFS, disease-free survival.

features related with high PD-L1 expression in TETs (Table 2). The seven studies mentioned earlier showed high PD-L1 expression associated with aggressive histology, such as in type B2/B3 thymoma or thymic carcinoma, although one study adversely identified significant association between high PD-L1 expression and the histology of thymoma rather than thymic carcinoma. Moreover, some studies found that high PD-L1 expression was significantly associated with advanced Masaoka-Koga stage. As such, PD-L1 is speculated to be particularly overexpressed in TETs with high malignant potential or in advanced unresectable cases that are considered sufficiently eligible for anti-PD-1/PD-L1 blockade therapy.

In terms of the prognostic impact of PD-L1 expression in TETs, the results slightly differ among the past studies. Katsuya et al. and Weissferdt et al. did not find any prognostic relevance of PD-L1 expression in TETs $(14,18)$. By contrast, Padda et al. found that high PD-L1 expression in TETs was associated with poor overall survival (OS) in sex- and age-adjusted analysis (13). The authors also determined significant correlation between high PD-L1 expression and poor disease-free survival, but not in OS, after complete resection in thymoma (15). By contrast, significant association was found between high PD-L1 expression and better OS in thymic carcinoma patients (16). Similarly, Arbour et al. also noted that high-risk TET cases (type B2/B3 thymoma and thymic carcinoma) with high PD-L1 expression had significantly better OS by using Kaplan-Meier methods (19). These conflicting results may be due to the study design. For instance, the authors considered that thymomas and thymic carcinomas should be individually analyzed because these tumors demonstrate distinct clinical behaviors. Thymomas are widely known to complicate autoimmune disorders represented by myasthenia gravis in approximately $25 \%$ of cases and pathologically involve immature $\mathrm{T}$ cells expressing terminal deoxynucleotidyl transferase (2). By contrast, thymic carcinomas are defined as tumors lacking immature $\mathrm{T}$ lymphocytes and never elicit autoimmune diseases (1). As such, the tumor microenvironment in thymoma and thymic carcinoma should be distinguished. In addition, thymic carcinoma also demonstrates significantly poor prognosis compared with thymoma (1). These factors should be considered when investigating the prognostic implications of PD-L1 expression in TETs, although it is often difficult owing to the rarity of the disease. Thus, the prognostic significance of PD-L1 expression in TETs should be determined only after analyzing thymoma and thymic carcinoma individually.

\section{PD-1 expression of tumor infiltrating lymphocytes (TILs) in TETs}

Four of the 7 aforementioned studies studying PD-L1 in TETs also assessed PD-1 expression of TILs in TETs. Katsuya et al. were the first to report PD-1 expression in TILs, but not in neoplastic cells in thymic carcinoma (14). Considering their cutoff value, $62 \%$ of thymic carcinoma was classified as high PD-1 expression; however, no prognostic relevance was detected. The authors examined 25 cases of thymic carcinomas and noted that PD-1 expression in TILs was not associated with PD-L1 expression, but increased PD-1-positive TILs indicated a poor prognosis. Meanwhile, Weissferdt et al. reported that PD-1-positive TILs were found in $23 \%$ of thymic carcinomas and $62 \%$ of thymomas according to their criteria. They also identified 
a significant association between increased PD-1-positive TILs and high stage in thymic carcinoma; nevertheless, the prognostic impact was not determined. Arbour et al. reported a significant association between high PD-1 expression and high/moderate glucocorticoid-induced tumor necrosis factor receptor (GITR) expression in highgrade TETs. GITR is a modulator of regulatory T-cells and a costimulatory molecule that has the potential to activate cytotoxic T-cells (22-25).

Despite these studies, the prognostic significance of PD-1 expression in TETs remains unclear. Theoretically, PD-1 expression in thymic carcinoma may have poor prognostic relevance due to the negative regulatory effect of cytotoxic T cells through PD-1/PD-L1 pathway. However, some authors determined mutually exclusive staining between PD-L1 and PD-1 expression in TETs, suggesting that inactivation of the PD-1/PD-L1 pathway may compromise the effectiveness of blockade therapy in this pathway $(14,17)$. This point should be further clarified in detail in future studies with additional measurements, such as double IHC.

\section{Immunotherapeutic agents currently approved for clinical administration in malignant tumors}

Several kinds of anti-PD-1, anti-PD-L1, and anti-CTLA-4 antibodies have been recently approved by the U.S. FDA for use in clinical settings (26). As of July 2017, the antiPD-1 antibodies nivolumab and pembrolizumab are available, which are both fully humanized IgG4 monoclonal antibodies against PD-1 receptor. Nivolumab has been approved for patients with metastatic melanoma, nonsmall cell lung cancer, renal cell carcinoma, squamous cell carcinoma of the head and neck, urothelial carcinoma, and classical Hodgkin lymphoma, while pembrolizumab has been approved for metastatic melanoma, non-small cell lung cancer, squamous cell carcinoma of the head and neck, urothelial carcinoma, classical Hodgkin lymphoma, and solid tumors with high microsatellite instability or mismatch repair deficiency. As anti-PD-L1 antibodies, atezolizumab, a humanized engineered monoclonal antibody of IgG1 isotype, is approved for clinical administration in patients with non-small cell lung cancer and urothelial carcinoma. Furthermore, anti-PD-L1 antibodies avelumab and durvalumab are also available. On the other hand, ipilimumab, a fully humanized anti-CTLA-4 monoclonal IgG1 antibody, is approved only for patients with malignant melanoma.

\section{Clinical availability of anti-PD-1/PD-L1 blockade therapy for TETs}

Some authors published sufficient therapeutic effects of PD-1/PD-L1 inhibitors for TETs in individual case reports, contributing in the promotion of clinical trials for advanced TETs $(27,28)$. Subsequently, some phase I clinical trials used PD-1/PD-L1 inhibitors for solid malignant tumors including TETs, and demonstrated acceptable clinical efficacy and safety of the drug despite the limited number of patients (29-31). However, severe systemic mucocutaneous toxicity requiring plasmapheresis caused by pembrolizumab in a patient with type B2 thymoma has been reported, suggesting the requirement of careful patient selection in using immunotherapeutic agents (27).

Some phase II trials evaluating the clinical availability of pembrolizumab for thymic carcinoma are currently in progress $(32,33)$. Giaccone et al. investigated 23 thymic carcinoma patients with disease progression after at least one line of chemotherapy and without autoimmune disease or other malignancy and found a response rate of $24 \%$; 1 patient achieved complete response, 4 achieved partial response, 9 achieved stable disease, and 7 had disease progression. The toxicity was reported to be well tolerated, and mild fatigue, fever, diarrhea, and rhinorrhea were the major side effects. However, serious autoimmune disorders developed in two patients; one patient experienced severe myositis and myocarditis that required pacemaker implantation and the other patient developed type I diabetes mellitus after treatment discontinuation. The other trials by Cho et al. targeted 33 TET patients (26 with thymic carcinomas and 7 with thymomas). The eligibility criteria included progression after platinum-based chemotherapy. Patients with active autoimmune disease that required systemic therapy within the past 1 year were excluded. The overall response rate was $24.2 \%$; 8 patients had partial response, 17 had stable disease, and 8 had progressive disease. In this study, various $\geq$ grade 3 immunological side effects were observed, including hepatitis, myocarditis, myasthenia gravis, thyroiditis, glomerulonephritis, colitis, and subcutaneous myoclonus.

These studies suggested that pembrolizumab can induce acceptable responses against TETs in clinical settings. However, future studies should be designed with extensive surveillance particularly focused on immunological adverse events. Remarkably, various autoimmune disorders of which the detailed pathogenesis remain uncertain are known to be complicated in thymoma, the most common of which is 
myasthenia gravis. These poorly understood autoimmune mechanisms may be associated with the development of severe immunological adverse events from PD-1/PD-L1 blockade. Although rare, a fatal case of myasthenia gravis caused by pembrolizumab has been reported (34). Therefore, attentive discussions by multidisciplinary experts are needed for the off-label use of PD-1/PD-L1 blockade agents for TETs.

\section{Concluding remarks}

Given the poor response of TETs to chemotherapeutic agents, the clinical administration of immunotherapy is a promising therapeutic alternative for advanced TETs. However, further careful evaluation of the efficacy and safety of immunotherapy for TETs is required. To optimize these drugs for refractory TETs, additional studies which evaluate clinical availabilities of immunotherapeutic drugs and characterize their basic mechanisms of action against immunotherapeutic targets are both urgently required.

\section{Acknowledgements}

None.

\section{Footnote}

Conflicts of Interest: The authors have no conflicts of interests to declare.

\section{References}

1. Travis WD, Brambilla E, Burke AP, et al. WHO classification of tumours of the lung, pleura, thymus and heart. 4th edition. Lyon: IARC, 2015:183-243.

2. Kondo K, Monden Y. Therapy for thymic epithelial tumors: a clinical study of 1,320 patients from Japan. Ann Thorac Surg 2003;76:878-84.

3. Venuta F, Anile M, Diso D, et al. Thymoma and thymic carcinoma. Eur J Cardiothorac Surg 2010;37:13-25.

4. Moon JW, Lee KS, Shin MH, et al. Thymic epithelial tumors: prognostic de-terminants among clinical, histopathologic, and computed tomography findings. Ann Thorac Surg 2015;99:462-70.

5. Ogawa K, Toita T, Uno T, et al. Treatment and prognosis of thymic carcinoma: a retrospective analysis of 40 cases. Cancer 2002;94:3115-9.

6. Yano M, Sasaki H, Yokoyama T, et al. Thymic carcinoma: 30 cases at a single institution. J Thorac Oncol 2008;3:265-9.

7. Girard N, Lal R, Wakelee H, et al. Chemotherapy definitions and policies for thymic malignancies. J Thorac Oncol 2011;6:S1749-55.

8. Schmitt J, Loehrer PJ Sr. The role of chemotherapy in advanced thymoma. J Thorac Oncol 2010;5:S357-60.

9. Liang SC, Latchman YE, Buhlmann JE, et al. Regulation of PD-1, PD-L1, and PD-L2 expression during normal and autoimmune responses. Eur J Immunol 2003;33:2706-16.

10. Brown JA, Dorfman DM, Ma FR, et al. Blockade of programmed death-1 ligands on dendritic cells enhances T cell activation and cytokine production. J Immunol 2003;170:1257-66.

11. Schreiber RD, Old LJ, Smyth MJ. Cancer immunoediting: integrating im-munity's roles in cancer suppression and promotion. Science 2011;331:1565-70.

12. Pardoll DM. The blockade of immune checkpoints in cancer immunotherapy. Nat Rev Cancer 2012;12:252-64.

13. Padda SK, Riess JW, Schwartz EJ, et al. Diffuse high intensity PD-L1 staining in thymic epithelial tumors. J Thorac Oncol 2015;10:500-8.

14. Katsuya Y, Fujita Y, Horinouchi H, et al. Immunohistochemical status of PD-L1 in thymoma and thymic carcinoma. Lung Cancer 2015;88:154-9.

15. Yokoyama S, Miyoshi H, Nishi T, et al. Clinicopathologic and Prognostic Implications of Programmed Death Ligand 1 Expression in Thymoma. Ann Thorac Surg 2016;101:1361-9.

16. Yokoyama S, Miyoshi H, Nakashima K, et al. Prognostic Value of Pro-grammed Death Ligand 1 and Programmed Death 1 Expression in Thymic Car-cinoma. Clin Cancer Res 2016;22:4727-34.

17. Marchevsky AM, Walts AE. PD-L1, PD-1, CD4, CD8 expression in neo-plastic and nonneoplastic thymus. Hum Pathol 2017;60:16-23.

18. Weissferdt A, Fujimoto J, Kalhor N, et al. Expression of PD-1 and PD-L1 in thymic epithelial neoplasms. Mod Pathol 2017;30:826-33.

19. Arbour KC, Naidoo J, Steele KE, et al. Expression of PD-L1 and other im-munotherapeutic targets in thymic epithelial tumors. PLoS One 2017;12:e0182665.

20. Philips GK, Atkins M. Therapeutic uses of anti-PD-1 and anti-PD-L1 anti-bodies. Int Immunol 2015;27:39-46.

21. Meng $X$, Huang $Z$, Teng F, et al. Predictive biomarkers in PD-1/PD-L1 checkpoint blockade immunotherapy. Cancer Treat Rev 2015;41:868-76. 
22. Shimizu J, Yamazaki S, Takahashi T, et al. Stimulation of CD25(+)CD4(+) regulatory T cells through GITR breaks immunological self-tolerance. Nat Immunol 2002;3:135-42.

23. McHugh RS, Whitters MJ, Piccirillo CA, et al. CD4(+) CD25(+) immunoreg-ulatory $T$ cells: gene expression analysis reveals a functional role for the glucocor-ticoidinduced TNF receptor. Immunity 2002;16:311-23.

24. Tone M, Tone Y, Adams E, et al. Mouse glucocorticoidinduced tumor ne-crosis factor receptor ligand is costimulatory for T cells. Proc Natl Acad Sci U S A 2003;100:15059-64.

25. Cohen AD, Schaer DA, Liu C, et al. Agonist anti-GITR monoclonal anti-body induces melanoma tumor immunity in mice by altering regulatory $\mathrm{T}$ cell sta-bility and intratumor accumulation. PLoS One 2010;5:e10436.

26. Tessmer MS, Flaherty KT. AACR Cancer Progress Report 2017: Harnessing Research Discoveries to Save Lives. Clin Cancer Res 2017;23:5325.

27. Zander T, Aebi S, Rast AC, et al. Response to Pembrolizumab in a Patient with Relapsing Thymoma. J Thorac Oncol 2016;11:e147-9.

28. Yang Y, Ding L, Wang P. Dramatic response to antiPD-1 therapy in a pa-tient of squamous cell carcinoma of thymus with multiple lung metastases. J Thorac Dis

Cite this article as: Yokoyama S, Miyoshi H. Thymic tumors and immune checkpoint inhibitors. J Thorac Dis 2018;10(Suppl 13):S1509-S1515. doi: 10.21037/jtd.2017.10.157
2016;8:E535-7.

29. Yamamoto N, Nokihara H, Yamada Y, et al. Phase I study of Nivolumab, an anti-PD-1 antibody, in patients with malignant solid tumors. Invest New Drugs 2017;35:207-16.

30. Rajan A, Heery C, Mammen A, et al. OA18.03 Safety and Clinical Activity of Avelumab (MSB0010718C; AntiPD-L1) in Patients with Advanced Thymic Epithelial Tumors (TETs). J Thorac Oncol 2017;12:S314-5.

31. Heery CR, O'Sullivan-Coyne G, Madan RA, et al. Avelumab for metastatic or locally advanced previously treated solid tumours (JAVELIN Solid Tumor): a phase 1a, multicohort, dose-escalation trial. Lancet Oncol 2017;18:587-98.

32. Giaccone G, Thompson J, Crawford J, et al. A phase II study of pembrolizumab in patients with recurrent thymic carcinoma. J Clin Oncol 2016;34:S8517.

33. Cho J, Ahn MJ, Yoo KH, et al. A phase II study of pembrolizumab for patients with previously treated advanced thymic epithelial tumor. J Clin Oncol 2017;35:S8521.

34. March KL, Samarin MJ, Sodhi A, et al. Pembrolizumabinduced myasthenia gravis: A fatal case report. J Oncol Pharm Pract 2018;24:146-9. 